\title{
Nasal Colonization of Staphylococcus aureus and their Antibiograms among School Children in Bharatpur, Nepal
}

\author{
Sanjib Adhikari, ${ }^{1}$ Sujan Khadka, ${ }^{1}$ Ashish Parajuli, ${ }^{1}$ Anjana $\mathrm{KC}^{1}$, Rajani Mishra, ${ }^{1}$ Puspa Kandel, ${ }^{1}$ \\ Ashik Tiwari ${ }^{1}$ \\ ${ }^{1}$ Birendra Multiple Campus, Bharatpur, Chitwan, Nepal.
}

\begin{abstract}
Background: Nasal carriage of Staphylococcus aureus has been linked to several community-acquired and nosocomial infections. The present study was undertaken to determine the prevalence and antibiotic susceptibility patterns of $S$. aureus colonizing the anterior nares of school children in Bharatpur, Nepal. Materials and Methods: In a cross-sectional study, 206 school children aged 3-15 years from fourteen different government schools in Bharatpur, Nepal were enrolled from January to May 2017. Prior to collecting sample, permission was sought from the administration of each school. All the participants were informed about the purposes of the study and the method of sample collection. Isolation of $S$. aureus and their antimicrobial susceptibility testing were performed by standard microbiological procedures. Results: $S$. aureus was isolated in $35(16.9 \%)$ cases of which $6(17.1 \%)$ were methicillin-resistant $S$. aureus (MRSA) and $7(20.0 \%)$ were multi-drug resistant (MDR). Prevalence of $S$. aureus and MRSA was higher in the age group $10-15$ years $(24.1 \%$ and $3.6 \%)$ compared to the age group $3-9$ years $(8.5 \%$ and $2.1 \%)(p=0.003)$. All the isolates were sensitive towards vancomycin and amikacin. Conclusions: Promotion of good hygienic practices among school going children can be suggested to abate the risk of spread and infections by $S$. aureus.
\end{abstract}

Keywords: antibiotic resistance; MRSA; nasal carriage; S. aureus; school children.

\section{INTRODUCTION}

Staphylococcus aureus is the most frequently faced species of clinical importance. ${ }^{1,2}$ S. aureus has also been identified as an important human pathogen. ${ }^{3}$ In addition $S$. aureus colonizes huge proportion of human population. ${ }^{4}$ About $20.0 \%$ to $30.0 \%$ of human population is estimated to be the long term carrier of $S$. aureus. ${ }^{5}$ The nose is considered to be the main ecological niche of this pathogen. ${ }^{6}$

Nasal carriage of $S$. aureus has been recognized as a risk factor for community-acquired and nosocomial infections. ${ }^{7}$ Nasal carriage among hospital personnel and patients is much greater (60.0\%-70.0\%) as compared to those among community carriers. ${ }^{8}$ Relationship between nasal carriage of $S$. aureus and MRSA and subsequent invasive staphylococcal infections is supported by genetic evidence. ${ }^{9}$ Young children have a higher carriage of $S$. aureus than adults. ${ }^{10}$

One of the seriously feared strains of $S$. aureus is methicillin-resistant $S$. aureus (MRSA), which shows resistant to virtually all $\beta$-lactam antimicrobials including penicillin, cephalosporin, carbapenem and monobactam. ${ }^{11}$ Treatment of infections caused by $S$. aureus, has become more difficult since the development of MRSA. Longer hospital stay, prolonged antibiotic usage and higher costs to treat for the infections caused by methicillinsusceptible $S$. aureus (MSSA) strains are associated with the infections caused by MRSA. ${ }^{12}$ Prevalence of MRSA ranges from $5.0 \%$ to $73.0 \%{ }^{13,14}$ Some strains of MRSA are epidemic in character (EMRSA). The drug of choice for treating MRSA infections is vancomycin. However, the emergence of vancomycin-intermediate or vancomycin-resistant strains of $S$. aureus has become a very great challenge for clinicians. ${ }^{15}$

The nasal carriage of $S$. aureus among the children in some cities in Nepal like Kathmandu and Pokhara have been previously assessed, however there's no any reports regarding nasal colonization of $S$. aureus among the children in Bharatpur. In this regard, the present study was conducted to determine the prevalence of nasal carriage of $S$. aureus in school

Correspondence: Mr. Sanjib Adhikari, Department of Microbiology, Birendra Multiple Campus, Bharatpur-10, Chitwan, Nepal. Email: sanadh26@gmail.com. Phone: +9779845891588. DOI: 10.3126/jcmsn.v14i4.19511. Article received: 2018-03-11. Article accepted: 2018-10-01. 
children in Bharatpur city and to observe the susceptibility of the recovered isolates against various antibiotics.

\section{MATERIALS AND METHODS}

\section{Study design and specimen collection}

A cross-sectional study was conducted among 206 school children aged 3-15 years from fourteen different government schools in Bharatpur, Nepal from January to May 2017. All the participants were informed about the purpose of the study and the procedure of sample collection. The study volunteers seemed healthy on physical examination and none of them had nasal abnormalities. Nasal swab specimens were collected by using sterile dry cotton-wool swabs, and both the anterior nares (left and right) were swabbed by rubbing the swab several times around the inside of each nostril and rotating the swab without any interruption.

\section{Specimen processing and identification}

All the nasal swabs were placed in buffered peptone water and then transported to and processed at Microbiology laboratory of Birendra Multiple Campus, Bharatpur, within an hour. The collected samples were inoculated on Blood Agar and Mannitol Salt Agar plates and incubated at $37^{\circ} \mathrm{C}$ for 24 hours. Culture-positive plates were further taken into macroscopic and microscopic examination. The organism identified as Grampositive cocci in bunch on Gram's staining was further confirmed by catalase and coagulase (slide and tube) tests. ${ }^{16}$

\section{Antibiotics susceptibility testing}

The antibiotic susceptibility testing was performed by using modified Kirby-Bauer disc diffusion technique against different antibiotics (HI-MEDIA, Mumbai, India) such as amikacin (30 mcg), cefoxitin $(30 \mathrm{mcg})$, ceftriaxone $(30 \mathrm{mcg})$, ciprofloxacin $(5 \mathrm{mcg})$, cloxacillin $(5 \mathrm{mcg})$, cotrimoxazole $(25 \mathrm{mcg})$, erythromycin $(15 \mathrm{mcg})$, gentamicin $(10 \mathrm{mcg})$, tetracycline $(30 \mathrm{mcg})$ and vancomycin $(30 \mathrm{mcg})$. Suspension of the isolates were adjusted to 0.5 MacFarland solutions and streaked on MHA plates. Antibiotics discs were placed on the streaked plates and incubated at $35^{\circ} \mathrm{C}$ for 18-24 hours and the interpretation was made based on Clinical Laboratory Standard Institute (CLSI 2016) guidelines. ${ }^{17}$ Cefoxitin $(30 \mathrm{mcg})$ disc was used for screening of MRSA. Isolates showing inhibition zone $\leq 21 \mathrm{~mm}$ around cefoxitin disc were identified as MRSA strain. S. aureus ATCC-25923 was used as positive control for the tests.

\section{Data analysis}

All the obtained data were analyzed using SPSS version 20. Chi-square test and Fischer's exact test were used to determine the possible risk factors for nasal carriage of $S$. aureus. P-value $\leq 0.05$ was considered to be statistically significant, at 5.0\% level of significance. Finally, all the obtained results were presented in tabulated form.

\section{Ethical approval}

Permission to conduct this research was granted from the Department of Microbiology, Birendra Multiple Campus. The purpose and procedure were clearly stated to the participants prior to specimen collection. The participants were selected only after they were sufficiently counseled and verbal informed consent was obtained from each participant and also from the school administration of each school. Only those children willing to participate in this study were included.

\section{RESULTS}

During the study period, 206 school children from 14 different government schools were screened for nasal carriage of $S$. aureus. The participants were categorized into two age groups: 3-9 years and 10-15 years. Ninety four $(45.6 \%)$ children belonged to 3-9 years of age and $112(54.3 \%)$ belonged to $10-15$ years of age. Altogether 99 (48.0\%) boys and 107 $(51.9 \%)$ girls were recruited in the study. Table 1 shows the sex and age-wise distribution of the study participants.

\begin{tabular}{|l|c|c|}
\hline \multicolumn{3}{|l|}{ Table 1. Sample distribution. } \\
\hline Category & Division & Size \\
\hline \multirow{3}{*}{ Sex } & Male & 99 \\
\cline { 2 - 3 } & Female & 107 \\
\cline { 2 - 3 } & Total & 206 \\
\hline \multirow{3}{*}{ Age } & $3-9$ years & 94 \\
\cline { 2 - 3 } & $10-15$ years & 112 \\
\cline { 2 - 3 } & Total & 206 \\
\hline
\end{tabular}

S. aureus was isolated from $35(17.0 \%)$ study subjects of which $6(17.1 \%)$ were screened as MRSA. Nasal carriage of $S$. aureus amongst boys was found to be $17.2 \%$ (17/99) whereas amongst girls was $16.8 \%(18 / 107)$. MRSA was detected from $2(2.0 \%)$ boys and $4(3.7 \%)$ girls. Prevalence of $S$. aureus and MRSA in the age group 3-9 years was $8.5 \%$ and $2.1 \%$ respectively whereas their prevalence in the age group $10-15$ years was $24.1 \%$ and $3.6 \%$ (Table 2 ).

The antibiotics susceptibility testing was performed on all the 35 isolates of $S$. aureus, out of which, 7 $(20.0 \%)$ showed resistance to two or more classes of 
Adhikari et al. Nasal Colonization of Staphylococcus aureus and their Antibiograms among..

\begin{tabular}{|c|c|c|c|c|c|c|c|}
\hline \multicolumn{2}{|c|}{ Study Characteristics } & \multicolumn{2}{|c|}{ S. aureus } & \multirow[t]{2}{*}{$\overline{\text { P-value }}$} & \multicolumn{2}{|c|}{ MRSA } & \multirow[t]{2}{*}{ P-value } \\
\hline & & $\begin{array}{c}\text { Present No. } \\
(\%)\end{array}$ & $\begin{array}{c}\text { Absent No. } \\
(\%)\end{array}$ & & $\begin{array}{c}\text { Present No. } \\
(\%)\end{array}$ & $\begin{array}{c}\text { Absent No. } \\
(\%)\end{array}$ & \\
\hline \multirow[t]{3}{*}{ Sex } & Male & $17(17.2)$ & $82(82.8)$ & \multirow[t]{3}{*}{0.947} & $2(2.0)$ & $97(98.0)$ & \multirow[t]{3}{*}{0.658} \\
\hline & Female & $18(16.8)$ & $89(83.2)$ & & $4(3.7)$ & $103(96.3)$ & \\
\hline & Total & $35(17.0)$ & $171(83.0)$ & & $6(2.9)$ & $200(97.1)$ & \\
\hline \multirow[t]{3}{*}{ Age } & $3-9$ years & $8(8.5)$ & $86(91.5)$ & \multirow[t]{3}{*}{0.003} & $2(2.1)$ & $92(97.9)$ & \multirow[t]{3}{*}{0.602} \\
\hline & $10-15$ years & $27(24.1)$ & $85(75.9)$ & & $4(3.6)$ & $108(96.4)$ & \\
\hline & Total & $35(17.0)$ & $171(83.0)$ & & $6(2.9)$ & $200(97.1)$ & \\
\hline
\end{tabular}

antibiotics tested (MDR). Among 35 S. aureus, 3 $(8.6 \%)$ were resistant towards ciprofloxacin and cefoxitin, $2(5.7 \%)$ were resistant towards ciprofloxacin and co-trimoxazole, $1(2.9 \%)$ was resistant towards ciprofloxacin and erythromycin, and $1(2.9 \%)$ was resistant towards cefoxitin, ciprofloxacin and erythromycin. All the MRSA isolates were sensitive towards tetracycline, gentamicin, vancomycin and amikacin whereas intermediately resistant towards cloxacilin. Higher percentage of resistance was observed amongst MRSA isolates against ciprofloxacin (66.0\%) compared to other antibiotics used. Similarly, 1 (16.1\%) MRSA isolate showed combined resistance towards ciprofloxacin and erythromycin. Vancomycin and amikacin were the most effective antibiotics used as all the isolates $(100.0 \%)$ were sensitive against these antibiotics whereas ciprofloxacin was found to be the least effective drug used as only $14(40.0 \%)$ isolates were resistant to it (Table 3). study by Soysal et al, in Turkish children (17.3\%) and by Okwa et al, in Nigerian school children $(18.3 \%){ }^{21,22}$ The present study reports fairly a higher nasal carriage of $S$. aureus in the school children in Bharatpur city in comparison to $11.7 \%$ nasal carriage among healthy school children in the capital city, Kathmandu. ${ }^{23}$ On the other hand, prevalence of $S$. aureus found in this study is lower than that of the study conducted in Pokhara, Nepal and in Jimma, Ethiopia which were $31.0 \%$ and $47.3 \%$ respectively. ${ }^{24,25}$ The variation in the prevalence of $S$. aureus among different studies could be due to the several factors such as difference in personal hygiene and lifestyle among different population characteristics, crowded living environment, direct skin to skin contact, hospitalization, sharing of individual objects etc. ${ }^{26}$

Among thirty-five $S$. aureus isolates, 6 (17.2\%) were found to be MRSA, in this study. The finding of this study is nearly similar to the study conducted

\begin{tabular}{|lllllll|}
\hline \multicolumn{7}{|l|}{ Table 3. Antibiotics susceptibility pattern of S. aureus and MRSA isolates. } \\
\hline Antibiotic & S. aureus & (N=35), $\mathbf{n}(\%)$ & \multicolumn{4}{c|}{ MRSA (N=6), $\mathbf{n}(\%)$} \\
& S & I & $\mathbf{R}$ & $\mathbf{S}$ & $\mathbf{I}$ & $\mathbf{R}$ \\
Cefoxitin & $29(82.9)$ & $0(0.0)$ & $6(17.1)$ & $0(0.0)$ & $0(0.0)$ & $6(100.0)$ \\
Cloxacillin & $15(42.9)$ & $20(57.1)$ & $0(0.0)$ & $0(0.0)$ & $6(100.0)$ & $0(0.0)$ \\
Ciprofloxacin & $17(48.6)$ & $4(11.4)$ & $14(40.0)$ & $1(16.7)$ & $1(16.7)$ & $4(66.7)$ \\
Tetracycline & $34(97.1)$ & $1(2.9)$ & $0(0.0)$ & $6(100.0)$ & $0(0.0)$ & $0(0.0)$ \\
Erythromycin & $20(57.2)$ & $9(25.7)$ & $6(17.1)$ & $3(50.0)$ & $2(33.3)$ & $1(16.7)$ \\
Gentamicin & $33(94.2)$ & $1(2.9)$ & $1(2.9)$ & $6(100.0)$ & $0(0.0)$ & $0(0.0)$ \\
Ceftriaxone & $27(77.1)$ & $8(22.9)$ & $0(0.0)$ & $2(33.3)$ & $4(66.7)$ & $0(0.0)$ \\
Cotrimoxazole & $25(71.4)$ & $6(17.1)$ & $4(11.4)$ & $4(66.7)$ & $2(33.3)$ & $0(0.0)$ \\
Amikacin & $35(100.0)$ & $0(0.0)$ & $0(0.0)$ & $6(100.0)$ & $0(0.0)$ & $0(0.0)$ \\
Vancomycin & $35(100.0)$ & $0(0.0)$ & $0(0.0)$ & $6(100.0)$ & $0(0.0)$ & $0(0.0)$ \\
\hline
\end{tabular}

\section{DISCUSSION}

$S$. aureus is considered to be a versatile pathogen, since it is one of the most common causes of nosocomial and community-acquired infections. ${ }^{18}$ The most consistent location for $S$. aureus is the anterior nares of a person. Although this colonization is a typical phenomenon, it may be a source for invasive infections. ${ }^{19} \mathrm{~S}$. aureus carriage is likely to decrease with age. ${ }^{20}$ In our study, $17.0 \%$ of the school children were found to be the nasal carriers of $S$. aureus. This result is in tune with the in Urmia, which reported the prevalence of MRSA as $14.9 \%$ among the $S$. aureus isolates in the preschool and school children of age below 14 years. ${ }^{27}$ Other studies in different countries show variation in MRSA colonization. The nasal colonization rates of MRSA reported in the same year in two different cities in Nepal were quite varying i.e. $5.2 \%$ in Kathmandu ${ }^{23}$ and $56.1 \%$ in Pokhara. ${ }^{24}$ A recent study in Iraq reported that nasal colonization of MRSA among the primary school children was $13.3 \%{ }^{28}$ 
In the present study, the rate of isolation of $S$. aureus in male school children was $17.2 \%$ and $16.8 \%$ in female school children. In a similar study conducted by Reta et al, nasal carriage of $S$. aureus was found to be $23.7 \%$ in male children and $17.3 \%$ in female children, which shows that the prevalence of $S$. aureus is more in female than in male, ${ }^{29}$ just like the result obtained from this study. On the other hand, a study conducted in Pokhara reported that the rate of isolation of $S$. aureus from male was $35.1 \%$ and $64.9 \%$ from female. ${ }^{24}$ Similarly, the study conducted in Urmia showed that the rate of isolation of $S$. aureus in male was $17.0 \%$ and in female was $23.5 \%{ }^{27}$ These results indicate that the nasal colonization of $S$. aureus in female is higher than the male counterparts which is in contrary to the findings of our study. The present study showed the nasal carriage of MRSA amongst male and female school children was $2.0 \%$ and $3.7 \%$ respectively. Our finding is similar to another study by Assafi et al, in Iraq who reported the prevalence of MRSA in male as 3.8\% and in female as $4.4 \%{ }^{28}$

Nasal carriage of $S$. aureus was also studied in two different age-groups: $3-9$ years and 10-15 years. In the study, $8.5 \%$ of the children belonging to the age group 3-9 years and $24.1 \%$ belonging to the age group 10-15 years were found to harbor $S$. aureus. The present study showed a higher colonization of $S$. aureus in the age group 10-15 years. A significant association was noted between the age group and the nasal colonization of $S$. aureus (pvalue 0.003 ). In a similar study conducted in Ethiopia, the children were divided into two different age groups: 6-9 years and 10-12 years and a higher prevalence of $S$. aureus was found in the age group 10-12 years (45.2\%) compared to the age group 6-9 years (36.4\%). ${ }^{29}$ In this study, nasal carriage of MRSA in 3-9 years age group was found to be $2.1 \%$ and its carriage in the age group 10-15 was found to be $3.6 \%$. Different studies show variations in the rate of colonization of MRSA. In Ethiopia, Reta et al, further reported colonization of MRSA was $12.7 \%$ and $15.4 \%$ among 6-9 years old children and 10-12 years old children respectively. ${ }^{29}$ According to a previous study, among 4-6 years age group of healthy children in Turkey, the nasal carriage of MRSA was $0.3 \%{ }^{30}$ While, among the children of $1-5$ years age in Philippines, $1.3 \%$ were the carriers of MRSA. ${ }^{31}$ Similarly, Dey et al, reported the carriers of MRSA as $10.2 \%$ among the children of $1-5$ years in India. ${ }^{32}$
In the antibiotic sensitivity testing, we observed that all the isolates were sensitive towards amikacin and vancomycin, which is similar to the findings reported by Habeeb et al, ${ }^{33}$ in Iraq and by Paulino et al, in Philippines. ${ }^{31}$ Higher number of isolates were resistant towards ciprofloxacin (40.0\%), followed by erythromycin $(17.1 \%)$, co-trimoxazole $(11.4 \%)$ and gentamicin $(2.9 \%)$. In a similar study at Ujjain, India, children of 1-6 years were studied. In this particular study, $4.0 \%$ of the total $S$. aureus isolates were resistant to vancomycin, $28.0 \%$ resistant to cotrimoxazole, $32.0 \%$ resistant to gentamicin, $41.0 \%$ resistant to tetracycline, $16.0 \%$ resistant to ciprofloxacin and $17.0 \%$ resistant to ceftriaxone. ${ }^{32}$ Emergence of MDR strains of $S$. aureus have been problematic to treat infections caused by them. This study revealed that $7(20.0 \%)$ of the $S$. aureus isolates were MDR. In a study in India, $11.0 \% S$. aureus showed combined MDR to ciprofloxacin and co-trimoxazole. ${ }^{32}$ In this study, MRSA strains showed resistance towards ciprofloxacin (66.7\%) and towards erythromycin (16.7\%). These findings are different than the study conducted by Reta et al, in Ethiopia, where none of the MRSA isolates were resistant towards these two antibiotics, ${ }^{29}$ whereas according to the results obtained from the study conducted by Rijal et al., in Pokhara, MRSA strains showed resistance towards these two antibiotics. ${ }^{24}$

\section{CONCLUSIONS}

The present study showed that the rate of nasal carriage of $S$. aureus was $16.9 \%(35 / 206)$ and MRSA nasal carriage prevalence was $2.9 \%(6 / 206)$. $S$. aureus colonization was higher in male children than in female children, while MRSA colonization was higher in female than in male. This study also revealed that the children of higher age are more prone to be the carriers of $S$. aureus and MRSA. The most effective antibiotics against the isolates were amikacin and vancomycin, while ciprofloxacin was found to be the least effective antibiotic. There is a growing urgency to promote activities in order to improve the hygienic behavior of school children in Bharatpur.

\section{ACKNOWLEDGEMENTS}

We are very thankful to the Department of Microbiology, Birendra Multiple Campus for providing us the laboratory facilities to conduct this study. We would also like to express our gratitude to the school Principals and all the participants for cooperating with us and helping us during sample collection. 


\section{REFERENCES}

1. Gotz F, Bannerman T, Schleifer K. The Genera Staphylococcus and Macrococcus. In F. Gotz, T. Bannerman, K. Schleifer, M. Dworkin, S. Falkow, E. Rosenberg, K.-H. Schleifer, E. Stackebrandt (Eds.). The Prokaryotes Berlin: Springer. 2006:5-75. https://doi.org/10.1007/0387-30744-3 1.

2. Brooks G, Carroll K, JS B. Jawetz, Melnick and Adelberg's Medical Microbiology. New York: The McGraw Hill Companies. 2010.

3. Rongpharpi S, Hazarika N, Kalita H. The prevalence of nasal carriage of Staphylococcus aureus among healthcare wokers at a tertiary care hospital in Assam with special reference to MRSA. Journal of Clinical and Diagnostic Research. 2013;7(2):257-260. https:// doi.org/10.7860/jcdr/2013/4320.2741.

4. Nilsson P, Ripa T. Staphylococcus aureus throat colonization is more frequent than colonization in the anterior nares. Journal of Clinical Microbiology. 2006;44:3334-3339. https:// doi.org/10.1128/jcm.00880-06.

5. Tong S, Davis J, Eichenberger E, Holland T, Fowler V. Staphylococcus aureus infection: epidemiology, pathophysiology, clinical manifestations, and management. Clinical Microbiology Reviews. 2015. 28(3):603-661. https://doi.org/10.1128/cmr.00134-14.

6. Wertheim H, Melles D, Vos M, van Leeuwen $\mathrm{W}$, van Belkum A, Verburgh $\mathrm{H}$, et al. The role of nasal carriage in Staphylococcus aureus infections. The Lancet Infectious Diseases. 2005;5:751-762.

7. Muge O, Zeynep B, Cem A. Nasal carriage of Staphylococcus aureus in healthy preschool children. Japanese Journal of Infectious Diseases. 2008;61:70-72.

8. Agraval S, Khan A, Srivastava A. Staphylococcal wound infection in surgical ward at Lucknow. Indian Journal of Medical Research. 2006;72(6):807-813.

9. Lo W, Wang C, Lin W, Wang S, Teng C, Huang $\mathrm{C}$, et al. Changes in the nasal colonization with Methicillin-resistant Staphylococcus aureus in children. Public Library of Science. 2010;5 (12):15791.

10. Tanaka Y, Adachi A, Ashimoto A. Drug resistant Staphylococcus aureus contamination in the ward environment. Kansenshogakn Zasshi. 2002;66:1270-1275. https:// doi.org/10.11150/

kansenshogakuzasshi1970.66.1270.

11. Kwon N, Park K, Jung W, Youn H, Lee Y, Kim $\mathrm{S}$, et al. Characteristics of Methicillin-resistant Staphylococcus aureus isolated from chicken meat and hospitalized dogs in Korea and their epidemiological relatedness. Veterinary Microbiology. 2006;117:304-312. https:// doi.org/10.1016/j.vetmic.2006.05.006.

12. Kumar P, Shukla I, Varshney S. Nasal screenig of healthcare workers for nasal carriage of coagulase positive MRSA and prevalence of nasal colonization with Staphylococcus aureus. Biology and Medicine, 2011; 3(2):182-186.

13. Diekema D, Pfaller M, Schmitz F, Smayevsky J, Bell J, Jones R, et al. Survey of infections due to Staphylococcus species: frequency of occurrence and antimicrobial susceptibility of isolates collected in the United States, Canada, Latin America, Europe, and the Western Pacific Region for the SENTRY Antimicrobial Surveillance Program, 1997-1999. Clinical Infectious Diseases 2001;32:114-132. https://doi.org/10.1086/320184.

14. Ankur B, Devjyoti M, Bernali P. Prevalence of nasal carriage of Mehicillin-resistant staphylococci in healthy population of Gangtok, East Sikkim. Journal of International Medical Sciences Academy, 21. 2008:191-193. https:// dx.doi.org/10.4103/0970-0218.58403.

15. Cosgrove S, Saroulas G, Perencevich E, MJ S, Karchmer A, Carmeli Y. Comparison of mortality associated with Methicillin-resistant and Methicillin-susceptible Staphylococcus aureus bacteremia: a meta-analysis. Clinical Infectious Diseases. 2003;36:53-56.

16. Baired D. Cluster Forming Gram-Positive Cocci. In D. Baired, J. Collee, A. Fraser, B. Marmion, \& A. Simmons (Eds.), Mackie and MaCartny Practical Medical Microbiology. Edinburgh, U.K.: Churchill Livingstone Limited. 1996:245-261.

17. Clinical and Laboratory Standards Institute. Performance standards for antimicrobial susceptibility testing. CLSI. Pennsylvania, USA. 2016.http://www.ljzx.cqrmhospital.com/ upfiles/201601/20160112155335884.pdf.

18. Rajbhandari R, Manandhar S, Shrestha J. Comparative study of MRSA and its antibiotics susceptibility pattern in indoor and outdoor patients of Bir Hospital. International Journal of Infection and Microbiology. 2003;1: 62-63.

19. Graham PI, Lin S, Larson E. A US populationbased survey of Staphylococcus aureus colonization. Annals of Internal Medicine 144, 2006:318-325. https://doi.org/10.7326/0003-4819144-5-200603070-00006.

20. Harputluoglu U, Egeli E, Sachin I, Oghan F, Ozturk O. (2005). Nasopharyngeal aerobic bacterial flora and Staphylococcus aureus nasal carriage in deaf children. International Journal of Pediatric Otorhinolaryngology. 2005;69(1):6974. https://doi.org/10.1016/j.ijporl.2004.08.005. 
Adhikari et al. Nasal Colonization of Staphylococcus aureus and their Antibiograms among..

21. Soysal A, Sahin H, Yagci A, Barlun I, Bakir M. The low rate of Methicillin-resistant Staphylococcus aureus in Turkish children. Japanese Journal of Infectious Diseases. 2006;59:195-196.

22. Okwaet M, Bamgbala S, Aborisade W. Prevalence of nasal carriage of communityassociated Methicillin-resistant Staphylococcus aureus (CA-MRSA) among healthy primary school children in Okada, Nigeria. Journal of Natural Sciences Research. 2012;2:2224-3186.

23. Joshi D, Shrestha S, Bomjan R, Paudel K. Nasal carriage of Staphylococcus aureus among healthy school children in Kathmandu. Nepal Journal of Science and Technology.2008;9139142. https://doi.org/10.3126/njst.v9i0.3177.

24. Rijal K, Pahari N, Shrestha B, Nepal A, Paudel $\mathrm{B}$, Mahato $\mathrm{P}$, et al. Prevalence of Methicillinresistant Staphylococcus aureus in school children of Pokhara. Nepal Medical College. 2008;10:192-195.

25. Kejela T, Bacha K. Prevalence and antibiotic susceptibility pattern of Methicillin-resistant Staphylococcus aureus (MRSA) among primary school children and prisoners in Jimma Town, Southwest Ethiopia. Annals of Clinical Microbiology and Antimicrobials. 2013;12 (11):1-11. https://doi.org/10.1186/1476-0711-12 -11 .

26. Braga E, Anguir-Alves F, de Freitas MF, de e Silva M, Correa T, Snyder R, et al. High prevalence of Staphylococcus aureus and Methicillin-resistant S. aureus colonization among healthy children attending public daycare centers in informal settlements in a large urban center in Brazil. BMC Infectious Diseases. 2014:14-538. https://doi.org/10.1186/1471-2334 -14-538.

27. Sadeghi E, Far A, Karamiyat M, Gazzavi A, Nikibakhsh, A, Naroozi M. Frequency of Methicillin-resistant Staphylococcus aureus nasal colonization among preschool and school children under 14 years old in Urmia. Journal of Urmia University Medical Sciences. 2017;27 (12):1041-1047. acadpub.umj.27.12.1041.

28. Assafi M, Polse R, Hussein N, Haji A, Issa A. The prevalence of Staphyoloccus aureus nasal colonization and its antibiotic susceptibility pattern amongst primary school pupils. Sciene Journal of University of Zakho. 2017;5(1):7-10. https://doi.org/10.25271/2017.5.1.291.

29. Reta A, Gedefaw L, Sewunet T, Beyene G. Nasal carriage, risk factors and antimicrobial susceptibility pattern of Methicillin-resistant Staphylococcus aureus among school children in Ethiopia. Jornal of Medical Microbiology and Diagnosis. 2015;4: $177 . \quad$ https:// doi.org/10.4172/2161-0703.1000177.

30. Ciftci H, Koken R, Bukulmez A, Ozdemir M, Safek B, Cetinkaya Z. Nasal carriage of Staphylococcus aureus in 4 to 6 age groups in healthy children in Afyonkarahisar, Turkey. Acta Paediatrica. 2007;96(7):1043-1046. https:// doi.org/10.1111/j.1651-2227.2007.00315.x.

31. Paulino C, Garcia R, Ong S. Staphylococcus aurus nasal carriage rates among children between oneto-five years in Barangay Pio Del Pilar, Makati city. Pediatric Infectious Disease Society of the Philippines. 2013;14(1);24-33.

32. Deyet S, Rosales-Klintz S, Shouche S, Pathak J, Pathak A. Prevalence and risk factors for nasal carriage of Staphylococcus aureus in children attending anganwaries (preschools) in Ujjan, India. BMC Research Notes.2013;9(6):265.https:// www.doi.org/10.1186/1756-0500-6-265.

33. Habeeb A, Hussien N, Assafi M, Al-Dabbgh S. Methicillin-resistant Staphylococcus aureus nasal colonization among secondary school students at Duhok city, Iraq. Journal of Microbiology and Infectious Diseases.2014;4(2):59-63. https:// doi.org/10.5799/ahinjs.02.2014.02.0128.

Citation: Adhikari S, Khadka S, Parajuli A, KC A, Mishra R, Kandel P, Tiwari A. Nasal Colonization of Staphylococcus aureus and their Antibiograms among School Children in Bharatpur, Nepal. JCMS Nepal. 2018;14(4):172-7. 